\title{
Moral Distress and Moral Courage in Everyday Nursing Practice
}

\author{
Ann Gallagher, PhD, MA, PGCEA, Bsc (Hons), RMN, SRN
}

\begin{abstract}
In this article the author examines the concepts of moral distress and moral courage within the context of nursing practice. Examples of challenging healthcare situations from the United Kingdom and Ireland are discussed in the light of the examination of these two concepts. The examples illuminate features of healthcare situations that need to be considered in relation to different organisational and cultural contexts. This requires an understanding of the complexity of clinical contexts and an appreciation of the fallibility and vulnerability of nurses and other practitioners. The goal of this article is to encourage healthcare organisations to create supportive structures and sensitive leadership that will enhance moral courage in the work setting.
\end{abstract}

Citation: Gallagher, A., (March 21, 2010) "Moral Distress and Moral Courage in Everyday Nursing Practice" OJIN: The Online Journal of Issues in Nursing Vol. 16 No. 2.

DOI: 10.3912/OJIN.Vol16No02PPT03

Keywords: moral distress, ethical climate, moral courage, nurses, unethical practice, organisations, clinical context

If people know what they should do, why don't they do it? Recently media and research in the United Kingdom (UK) and Republic of Ireland have addressed poor practice in healthcare. In one situation an Irish inquiry team investigated an unacceptably high rate of postpartum hysterectomies at the Lourdes hospital over a 25 -year period. The team commented that they had "difficulty understanding why so few had the courage, insight, or integrity to say, this is not right" (Harding Clark, 2006 p. 316). In another situation a UK inquiry into care detailed harrowing examples of patient neglect and high mortality rates at a Mid Staffordshire National Health Service Trust Hospital. The author of this inquiry report suggested that staff shortages, particularly amongst nurses, contributed to this substandard care. He observed that staff who spoke out felt ignored and that many staff were deterred from speaking out by fear and bullying (Francis, 2010). A third situation involved a survey by the Royal College of Nursing (RCN). Over 2,000 UK nurses participated in this survey. The RCN reported that $70 \%$ of the nurses sometimes left work feeling distressed and $11 \%$ always left work feeling distressed because they couldn't deliver the kind of dignifying care they knew they should provide (

...nursing practice does not take place in a vacuum and is not solely the responsibility of individual nurses at the bedside. So how should we think of all of these examples? Do people know what the right thing to do is? If people know what they should do, why don't they do it? Is it because they can't do the right thing because of individual, organisational, or cultural factors? Or that they could, but won't do the right thing? This article aims to explore some of the key concepts and factors that contribute to nurses and others doing or not doing the right thing in professional practice. Insights from philosophical and empirical work on the concept of moral distress help us to understand staff difficulties in this area. An exploration of the concept of moral courage supports the role of virtues in operationalising professional values. What becomes clear is that nursing practice does not take place in a vacuum and is not solely the responsibility of individual nurses at the bedside (micro level). 
Organisational (meso level) and political (macro level) factors may also influence the care provided. It will be argued that responses to moral distress and to unethical practice involve engagement with professional and organisational values and an aspiration for the organisation to become a virtuous organisation. The three examples introduced above will be revisited near the end of this article.

\section{Introduction to Moral Distress}

Moral distress is an evolving and contested concept; it has been described as a major problem in the nursing profession (Corley 2002). Jameton (1984) is credited with first identifying the concept of moral distress. He described moral distress as feelings that are painful, and as a psychological imbalance or disequilibrium that occurs when nurses finding themselves in situations where they feel unable to do the right thing. Jameton explained that moral distress arises when one knows the right thing to do, but institutional constraints make it nearly impossible to pursue the right course of action.

\section{Description of Moral Distress}

An extensive literature review by Schluter, Winch, Holzhauser, and Henderson (2008) has provided valuable insights regarding the definition, causes, influences, and effects of moral distress. Schluter et al. described moral distress as:

...an emotion that is expressed when the moral complexity of a situation is not leading to a resolution, thereby having the potential to cause harm to the individual nurse [...] painful feelings and associated mental anguish as a result of being conscious of a morally appropriate action, which, despite every effort, cannot be performed owing to organisational or other constraints (p. 306).

The context in which nurses work is an important consideration. Corley, Minich, Elswick, and Jacobs $(\underline{2005})$ referred to the ethical dimension of the work environment as characterised by aspects of an organisation that promote or inhibit professional nursing practice and, referring to the work of McDaniel (1997), where ethical values guide behaviours, including the setting of priorities that provide for the ethical treatment of patients.

Instruments have been developed to measure this phenomenon of ethical climate (McDaniel, Olson 1998) and research has been conducted to examine the correlation between moral distress and ethical climate. Pauly, Varcoe, Storch, and Newton (2009) found an inverse correlation between these two concepts, that is, better scores on moral climate (a more positive moral climate) correlated with less moral distress.

\section{Causes of Moral Distress}

The causes of moral distress identified in the literature review by Schluter et al. (2008) are threefold: poor-quality and futile care, unsuccessful advocacy, and raising unrealistic hope. In relation to the first cause, nurses have reported moral distress as a result of understaffing resulting in the quality of care being compromised and errors occurring. Their involvement in the provision of care they considered futile, or with no benefit to the patient, was also a contributory factor. Advocating unsuccessfully for patients was also found to be associated with moral distress. These advocacy situations were characterised both by confrontation with physicians, resulting in nurses feeling unable to achieve the best outcome for patients, and also by perceptions of lack of respect from 
physicians. Unrealistic hope arises when patients or families are given inaccurate or incomplete information regarding treatment outcomes and prognosis; in these situations the approach taken by the medical staff may result in nurses feeling ethically compromised and distressed.

\section{Factors Associated With Moral Distress}

Staff educational level and peer support have been found to influence moral distress. Staff educational level and peer support have been found to influence moral distress. The literature review by Schluter et al. (2008) identified a significant relationship between educational level/experience and moral distress, suggesting both that nurses with more education and experience have higher levels of moral distress and also that ethics education may improve coping strategies. Schluter et al. (2008) noted that coping strategies are also bolstered by good peer support, in that "the empathic and educated understanding provided by nursing peers who understand the surrounding circumstances was found to be most beneficial" (p. 315).

Additionally moral distress has been found to affect the health of nurses and their provision of care, job satisfaction, and retention. Nurses who experience moral distress have reported physical symptoms, such as headache, neck pain, and stomach problems. Psychological and emotional symptoms include anger, guilt, depression, frustration, and feelings of reduced self-worth. They may also withdraw from family and friends (Schluter et al., 2008)

\section{The Effects of Moral Distress}

Schluter et al. (2008) reported that the picture is mixed regarding the impact of moral distress on the provision of care, with some findings suggesting that it does not impact negatively on care and others suggesting that it results in nurses withdrawing from patients. Schluter and colleagues, however, did find that the evidence relating to job satisfaction and moral distress is less ambiguous in that moral distress was noted to reduce job satisfaction. This was attributed to nurses delivering care they consider unwarranted or futile and about which they were not consulted. These authors also found evidence that moral distress results in nurses leaving their jobs or the profession.

\section{Further Research}

The preceding discussion suggests that although a good deal is known about moral distress in nursing, under-researched areas remain. Writing in 2002 Corley, one of the pioneers of moral distress research and the developer of the Moral Distress Scale, pointed out that previous research has been "limited to instrument development and testing, descriptive findings and only one intervention study" (p. 648). Some work has explained the relationship between moral distress and the ethical climate of the organisation; yet questions regarding this relationship remain. The literature review by Schluter and colleagues (2008) indicated, for example, that we need to learn more about the impact of moral distress on the provision of care and the impact of education on moral distress. Research is also needed to identify strategies that will enable a constructive response to moral distress.

Thus far, there has been discussion of the meaning and implications of moral distress in nursing. There is evidence that nurses may experience distress and feel traumatised by situations in which they believe they are unable to do the right thing. The next section discusses the role of moral courage in supporting ethical nursing practice. 


\section{Introduction to Moral Courage}

It has been said that courage is the most admired of the virtues, that bravery is esteemed, and that cowardice is despised (Comte-Sponville, 2002). It is not difficult to think of current and past exemplars of courage. Lachman (2009) discussed two healthcare professionals who "epitomise the virtue of moral courage" (p. 3), namely, Albert Schwietzer and Florence Nightingale. A previous British Prime Minister, Gordon Brown (2007), discussed eight 'portraits' of courage. Some of these leaders have made significant contributions to healthcare, including Edith Cavell and Cicely Saunders. Edith Cavell sacrificed her life to save others during the First World War; Cicely Saunders set up St Christopher's hospice that continues to deliver high quality, end-of-life care today. Other portraits discussed by Brown have made significant contributions to world politics, peace, and human rights, including Nelson Mandela, Martin Luther King, and Aung San Suu Kyi.

Numerous healthcare situations call for courage. Examples include: breaking bad news regarding a poor prognosis, challenging a colleague who appears incompetent, delivering care to an infectious patient, confronting an angry relative, and raising concerns about unethical practice. What is common to all of these situations is the fear that may be experienced as the practitioner considers the cost of the action and the consequences of a particular intervention or of getting it wrong. There may be fear of an extreme emotional reaction, of violence, of contamination, of negative reactions from colleagues, or of losing one's job. Such fears may inhibit nurses and other practitioners from acting ethically. Lachman (2009) cited Albert Einstein as saying, "the world is a dangerous place, not because of those who do evil, but because of those who look on and do nothing" (p. 3). As discussed above, organisational constraints may cause nurses to feel as though they lack the courage to do the right thing or raise concerns about poor standards of care. This section discusses courage as a professional virtue and offers strategies that are likely to support courage in healthcare organisations.

\section{Courage as a Professional Virtue}

...one can 'go wrong' regarding courage, either by demonstrating too much courage or too little courage. One of the earliest accounts of courage has been provided by the Greek philosopher, Aristotle (1976 edition), who described it as the "right attitude towards feelings of fear and confidence" (p. 1115a-29). Although courage is considered a virtue, one can 'go wrong' regarding courage, either by demonstrating too much courage or too little courage. Virtue is straddled by two vices, one of excess (foolhardiness or rashness) and one of deficiency (cowardice). Nurses and others who are courageous in their everyday activities require professional wisdom, an intellectual virtue that ensures they demonstrate the right response to the fears they encounter (Banks \& Gallagher, 2009). Aristotle has explained that courage is developed by habituation. This means that nurses and others become courageous by doing courageous things, by getting into the habit of acting courageously. However, courage is also a reflective activity requiring self-scrutiny and learning from the feedback and role modelling of others. It is not necessarily the case, however, that a person will be courageous at all times and in all ways. It may be, as Adams (2006) has suggested, that individuals will have 'modules' of courage rather than composite courage. Modules of courage enable a nurse to respond courageously to some, but not all of the situations that inspire fear.

...even the most morally courageous staff may fear to speak up. The relationship between moral courage and moral distress is not straightforward. It is tempting to say that if nurses have sufficient 
moral courage they need not experience moral distress. Sufficient moral courage would enable them to speak up and challenge unacceptable practices and policies. However, given that organisations are not always supportive and do not always react appropriately, but rather may act defensively to concerns about standards of care raised by conscientious practitioners, even the most morally courageous staff may fear to speak up. A discussion of the three situations presented in the introduction can help to appreciate the complexity and challenges of preventing and stopping unethical practices.

\section{Factors that Support Moral Courage: An Examination of Three Examples}

The first situation described above related to unethical practices over a 25-year period at an Irish hospital whereby an unacceptably high number of peripartum hysterectomies were carried out (Harding Clark, 2006). The hospital had a Catholic ethos and was run by nuns with many locally trained staff. The practices continued over many years until two midwives, who had trained elsewhere, exposed the practices. The obstetrician and gynaecologist who had conducted most of the operations was eventually struck off the professional register. Readers may empathise with the Irish team who had difficulty understanding why so few had the courage to say that this was not right (Harding Clark, 2006). The inquiry report stated:

We uncovered a complex story, and many strands remain tangled in the personalities of the participants and the difficult relationship between religious beliefs and human reproduction overlaid with a sense of intense loyalty to the Maternity Unit. It is a story set in a time of unquestioning submission to authority, whether religious or civil, when nurses and doctors were in abundant supply and permanent jobs were few and treasured. The MMMs [the Medical Missionaries of Mary, an order of Catholic nuns] ran a very ordered hospital in an austere and dedicated manner (Harding Clark, 2006, p. 29).

It is all too easy to blame individuals for their inaction without taking into account the complexity of the situation as suggested in this quotation. A feminist analysis of this 'Irish Hysterectomy Scandal' by McCarthy, Murphy, and Loughrey (2008) examined the role and impact of 'deeply gendered asymmetries' related to power and privilege that existed in this situation. It is difficult to reduce explanations for non-action and silence on the part of the mostly female midwives and nurses to a lack of courage or integrity. Rather a variety of environmental factors contributed to their behaviours.

The Chairman of the Inquiry into the care at a Mid Staffordshire NHS Foundation Trust Hospital (Francis, 2010) pointed out, in his conclusion, the hospital was 'preoccupied' with cost cutting and other processes. This resulted in the Trust's losing sight of its responsibility to provide safe care. Francis added "It is now clear that some staff did express concern about the standard of care being provided to patients. The tragedy was that they were ignored and worse still others were discouraged from speaking out" (Paragraph 6). The report details many examples of care deficits that must be directly attributed to the ethical and professional failings of nurses and other professionals. However, again, it is important to understand that the organisational culture or climate within which these practitioners were working was a climate in which financial and other considerations were prioritised over ethical considerations and the well being of patients and staff. 
It is overly simplistic and misleading to blame nurses and others for failing to demonstrate moral courage at the individual level. Rather explanations and responses need to be provided at all three levels (micro, meso, and macro). The third example related to findings from a Royal College of Nursing survey ( $\underline{\mathrm{RCN}, 2008)}$. This survey assessed nurses' views of dignity in care, considering specifically what promotes dignity, what diminishes it, and what is the nurses' experience of delivering dignifying care. Many nurses reported that they left work feeling distressed because they couldn't deliver the kind of dignifying care they knew they should. The obstacles to, and enablers supporting, the delivery of dignified care related to individual or micro-level factors (the attitudes and behaviour of people); organisational or meso-level factors (organisational culture, leadership, resources, and the physical environment), and political or macro-level factors (government targets relating to waiting times).This report also highlighted the complex mix of factors that can contribute to moral distress in healthcare. It is overly simplistic and misleading to blame nurses and others for failing to demonstrate moral courage at the individual level. Rather explanations and responses need to be provided at all three levels (micro, meso, and macro).

\section{Conclusion}

The situations discussed in this article illustrate that particular organisational and cultural contexts have a significant impact on the freedom practitioners feel to demonstrate moral courage by raising concerns regarding activities and decisions in the organization. Examples of moral courage on the part of nurses are seen wherever nursing is practiced. An evaluation of the RCN dignity campaign in the UK, for example, highlighted many examples of innovative and inspiring practices led and implemented by nurses (Baillie \& Gallagher, 2010). There continues to be ample evidence, however, that nurses do not always feel they can do the right thing in their everyday practice. In some instances, this may be because they lack virtues, such as moral courage, wisdom, and integrity, virtues required to speak up and bring about change. In other instances, however, when organisational cultures are defensive, unsupportive, and potentially punishing, the virtue of courage may not be adequate to change a situation. I suggest that what is required is that organisations themselves also need to embrace the virtues of moral courage, wisdom, and integrity. They need to become virtuous organisations listening to and valuing patients, families, and staff. They need to have policies in place so that concerns and reports of poor practice are responded to quickly and professionally. They need to commend those who raise concerns rather than trying to silence them. They need to invest in leaders who will role model and take forward an ethical agenda. Strategies suggested by Lachman (2009), such as increasing organisational accountability and developing organisational ethics committees to facilitate ethical discourse and decision making, can help nurses to demonstrate moral courage by speaking up to make things right.

I suggest that what is required is that organisations themselves also need to embrace the virtues of moral courage, wisdom, and integrity. To return then to the questions in the introduction: Do people know what the right thing to do is? If people know what they should do, why don't they do it? Is it because they can't do it because of individual or organisational factors? Or that they could but won't do it? This article has discussed key concepts, including moral distress, moral courage, and ethical climate, that enable nurses and others to do, or not do the right thing in professional practice. Insights from philosophical and empirical work on the concept of moral distress help us to understand the challenges of doing the right thing. What should become clear is that the meaning of, and inter-relationships amongst, the concepts discussed in this article require reflection in 
relation to specific healthcare organisational and cultural contexts. It is important to learn from research relating to moral distress and ethical climates. However, it is equally important to engage in critical self-scrutiny, to invite feedback on the quality of our own professional practice, and to engage in dialogue with colleagues we respect regarding how things can be improved. The message, that doing the right thing is an organisational and a political as well as an individual responsibility, is one that needs to be emphasised.

\section{Author}

\section{Ann Gallagher, PhD, MA, PGCEA, Bsc (Hons), RMN, SRN}

E-mail: A.Gallagher@surrey.ac.uk

Dr. Gallagher is Reader in Nursing Ethics and Director of the International Centre for Nursing Ethics at the University of Surrey in Guildford, Surrey, United Kingdom. She trained as a state registered nurse in Belfast, Northern Ireland, and as a registered mental health nurse in Epsom, Surrey, United Kingdom. Her baccalaureate degree was a combined degree in philosophy and health studies. She holds a post-graduate certificate in the education of adults. Dr. Gallagher has been working in applied ethics for more than two decades and currently serves as the Editor of the Journal Nursing Ethics. Her current research interests include dignity in care, virtue ethics, and explanations for inadequate care and low staff morale.

\section{References}

Adams, R. (2006). A theory of virtue: Excellence in being for the good. Oxford: Oxford University Press.

Aristotle. (1976 Edition). The Nichomachean ethics of Aristotle, trans. Sir D Ross. London: Oxford University Press.

Baillie, L., \& Gallagher, A. (2010). Evaluation of the Royal College of Nursing's 'Dignity: at the heart of everything we do' campaign: Exploring challenges and enablers Journal of Research in Nursing, 15(1) pp.15-28.

Banks, S., \& Gallagher, A. (2009). Ethics in professional life: Virtues for health and social care. Basingstoke: Palgrave MacMillan.

Brown, G. (2007). Courage: Eight portraits. London: Bloomsbury Publishing.

Comte-Sponville, A. (2002). A short treatise on the great virtues: The uses of philosophy in everyday life. London: William Heineman.

Corley, M.C. (2002). Nurse moral distress: A proposed theory and research agenda Nursing Ethics, 9(6), 636-650.

Corley, M.C., Minick, P., Elswick, R.K., \& Jacobs, M. (2005). Nurse moral distress and ethical work environment. Nursing Ethics, 12(4), 381-390. 
Francis, R. (2010). Final report of the independent inquiry into care provided by Mid Staffordshire NHS Foundation Trust. Retrieved January 15, 2011 from www.midstaffsinquiry.com/pressrelease.html.

Harding Clark, M. (2006). The Lourdes Hospital inquiry - An inquiry into postpartum hysterectomy at Our Lady of Lourdes Hospital, Drogheda. The Stationary Office, Dublin. Retrieved January 15, 2011 from www.dohc.ie/publications/lourdes.html.

Jameton, A. (1984). Nursing practice: The ethical issues. London: Prentice-Hall.

Jameton, A. (1993). Dilemmas of moral distress: Moral responsibility and nursing practice. AWHONNS Clinical Issues Perinatal Womens Health Nursing, 4, 542-551.

Lachman, V.D. (2009). Ethical challenges in health care: Developing your moral compass. New York, NY: Springer Publishing Company.

McCarthy, J., Murphy, S., \& Loughrey, M. (2008). Gender and power: The Irish hysterectomy scandal. Nursing Ethics, 15(5), 642-655.

McDaniel, C. (1997). Development and psychometric properties of the ethics environment questionnaire. Medical Care, 35, 901-914.

Olson, L. (1998). Hospital nurses' perceptions of the ethical climate of their work setting. Image Journal of Nursing Scholarship, 30, 345-49.

Pauly, B., Varcoe, C., Storch, J., \& Newton, L. (2009). Registered nurses' perceptions of moral distress and ethical climate. Nursing Ethics, 16(5), 561-573.

Royal College of Nursing. (2008). Defending dignity: Challenges and opportunities for nursing. London: Royal College of Nursing. Retrieved from www.rcn.org.uk/ data/assets/pdf file/0011/166655/003257.pdf.

Schluter, J., Winch, S., Holzhauser, K., \& Henderson, A. (2008). Nurses' moral sensitivity and hospital ethical climate: A literature review. Nursing Ethics,15(3), 304-321.

(C) 2011 OJIN: The Online Journal of Issues in Nursing

Article published March 21, 2011 